\title{
CARCINOMA OF THE ENDOMETRIUM
}

\author{
By Braithwaite Rickford, M.D., B.S.(Lond.), F.R.C.S., M.R.C.O.G. \\ Obstetric Physician, St. Thomas's Hospital; Surgeon, Chelsea Hospital for Women
}

Carcinoma of the body of the uterus occurs principally in patients in the post-menopausal years, over half the cases arising between the ages of 50 and 60 , whereas with malignant disease of the cervix at least half arise before the cessation of menstruation. It is, however, important to realize that about 20 per cent. are found before the climacteric. This disease is less common than carcinoma in the cervix, the relationship being in the neighbourhood of three cervices to one body. The other notable comparison between these two conditions is the parity of these patients. One-third of the patients developing carcinoma of the body have not at any time been pregnant, whereas with the cervical group about one in ten are nulliparous.

\section{Aetiology}

Very little is known about the causes or predisposing conditions. There is little doubt that cystic hyperplasia is frequently an associated abnormality either as a previous condition or coincidental with malignancy. It has also been described on many occasions when a granulosa celled tumour is found in the ovary. These facts suggest that if the mucous membrane of the uterus has been subjected to prolonged oestrogen stimulation there is a greater risk of malignancy supervening. Whether the prolonged use of natural or synthetic oestrogens therapeutically can have the same effect has not been proved; such cases, however, do occur not infrequently. Finally, the use of radium to bring about an artificial menopause has been thought to predispose to malignancy at a later date. It is probable that when this has occurred unrecognized carcinoma has been present at the time of the insertion of the radium.

In view of this connection between endometrial hyperplasia and carcinoma, the use of oestrogens in the control of the menopausal syndrome and postmenopausal changes in the genital tract should be carefully controlled. These hormones, synthetic and otherwise, should only be prescribed for short periods and then only in such dosage that withdrawal bleeding is not produced.

\section{Pathology}

The vast majority of cases are basically columnar $\frac{\text { o }}{8}$ carcinoma. The pattern varies from a markedlye adenomatous type, ' adenoma malignum,' through a highly differentiated papilliferous type, to a $\mathcal{O}$ grossly anaplastic infiltrating growth. Squamous $\underset{\omega}{\omega}$ metaplasia is not infrequent giving rise to an + adeno-acanthoma.

Invasiveness also is extremely variable, in some cases spread into the uterine wall may be very early, whereas with others a large area of the endo- $\vec{\longrightarrow}$ metrium may be involved without any spread to muscle.

Macroscopically the disease may be ulcerated, $\stackrel{\oplus}{\stackrel{\oplus}{\rightarrow}}$ warty or grossly proliferating, filling the whofe o cavity of the uterus. It may be limited in area widely distributed, reaching at times as low as to internal os. Actual involvement of the canal of the cervix is unusual and such cases are usually classified separately. The size of the uterus may be very small, or moderately enlarged, and occasion- $\mathbb{D}$ ally, if the canal is obstructed with the formation of a haematometra, it may reach the size of a five $\frac{0}{3}$ month pregnancy.

Associated lesions are not infrequently present in the uterus. Fibroids are found in about a quarter of the cases. This fact in the premeno- $\frac{3}{3}$ pausal patient is of vital importance as the symptoms may be considered to be due to these rather 3 . than malignancy, which without a curettage may lead to inadequate surgical treatment.

The disease spreads by direct infiltration into o the uterine muscle which, being relatively thick and not in direct relationship with other organs, 음 tends to limit the disease for a considerable time. Later the outer surfaces become involved with $\sigma$ spread to the peritoneal cavity, omentum and at $N$ times the intestinal tract.

Lymphatic spread again is relatively late, $\frac{\omega}{\sigma}$ normally ascending along the ovarian vessels to the para-aortic glands. Occasionally the inguinal glands may be involved through the lymphatics of the round ligaments. Finally, a point which is ${ }_{7}^{+}$ possibly not sufficiently recognized, if the lower ${ }^{-}$ third of the endometrium is involved, spread may 
occur in exactly the same manner as carcinoma of the cervix, to the paracervical and iliac glands: This fact may well be the answer to the local recurrences after simple hysterectomy, and also to the retrograde secondaries found in the region of the vulva and lower third of the vagina.

Involvement of the ovaries is not infrequent. This may occur either by spread from the peritoneal surface of the uterus or by lymphatic permeation or emboli from the uterine fundus or possibly through the lumen of the tubes. Such deposits in the ovaries are frequently small and are found when no other gross evidence of spread has occurred, either in the peritoneal cavity or in the Fallopian tubes. It is probable, therefore, that they arise from direct lymphatic permeation rather than emboli which would be expected to involve the regional glands as well. This view is also supported by the fact that such cases even with considerably enlarged ovaries may survive for many years after surgical treatment.

\section{Symptoms}

Post-menopausal bleeding is the classical symptom. This may not be more than the slightest staining or it may be severe. It tends to occur relatively late so that when the loss is heavy quite extensive changes may be found. Preceding the bleeding some patients may notice an increased mucous discharge which is slightly discoloured. Pain is unusual until the peritoneal surface is involved unless the uterine cavity becomes distended with blood.

When carcinoma arises before the cessation of menstruation bleeding again is the major symptom. This may be an irregular intermenstrual loss or the timing may be similar to that of a metropathia or, more rarely, a regular menorrhagia is the only notable change.

\section{Diagnosis}

The diagnosis is established by a full endometrial curettage, which is essential in all patients who complain of post-menopausal bleeding, however slight. In many of these cases other abnormalities may be demonstrated, such as a cervical polyp, senile cervico-vaginitis or a caruncle, which might well give rise to the bleeding-but such conditions are relatively common and are frequently found in association with endometrial growths. They should never be accepted as the cause of this symptom until curettage has excluded malignancy.

Again irregular bleeding in the pre-menopausal years when it has occurred on more than two or three short occasions should never be allowed to continue without a thorough clearance and micro- scopical examination of the tissues from the uterus.

The use of the biopsy curette for removing small strips of endometrium in making a diagnosis is indefensible when dealing with the possibilities of carcinoma.

Vaginal smears examined for clumps of malignant cells at times may confirm a suspicion of endometrial growth, but more often than not nothing definite will be found, so that this method of diagnosis unlike its use with cervical growths cannot be relied upon to the exclusion of curettage.

Finally, with a patient who is known to have been taking oestrogens, if bleeding continues or recurs after discontinuing these drugs for two months, curettage is essential. If, however, there is no further loss, further investigation is unnecessary. Such patients at the time of oestrogen withdrawal bleeding frequently notice a congested feeling in the pelvis and slight pain of a dysmenorrhoeic type at the time of the bleeding which is unusual when carcinoma is the cause.

\section{Treatment}

Three main methods of treatment are in current use: (I) Total hysterectomy and bilateral salpingooophorectomy. (2) The same operation preceded by a large dose of intra-uterine radium six weeks previously, or by radiotherapy to the uterus with the same time interval. (3) Intra-uterine radium? alone, with hysterectomy later if the disease recurs in the uterus. The first is the method usually chosen in this country in suitable patients. The second is becoming recognized as the standard method in the United States of America, while the third has been adopted for all cases treated in

\section{Results of Treatment}

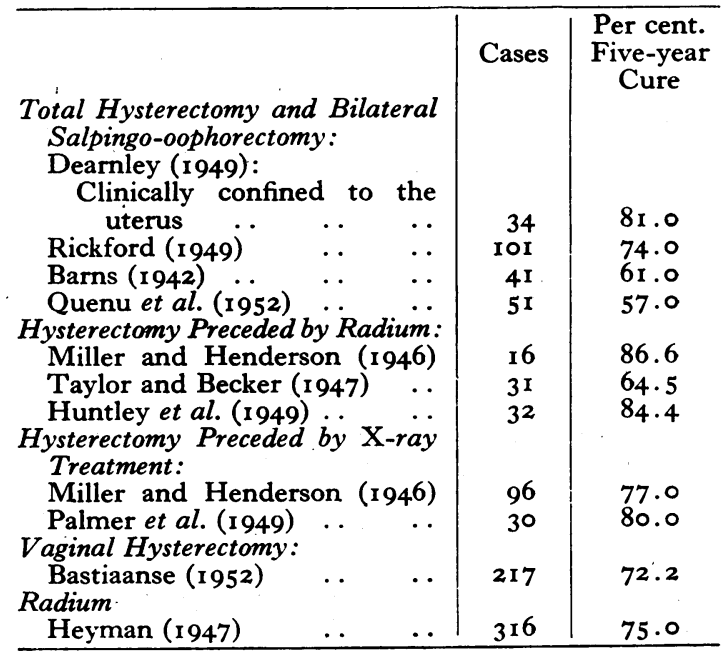


Stockholm by Professor Heyman and his successors.

Before describing these methods and the results obtained, there are various factors of general importance to be considered.

Many patients suffering from this disease are of advancing years, and may be suffering from various degenerative conditions of the cardiovascular system which make surgical treatment hazardous. Diabetes is not uncommonly a complicating factor. Chronic respiratory disease is often present or simple gross obesity, all of which increase the risks of a major abdominal operation. There is naturally, therefore, a group of patients who are better treated by radiation than by surgery.

\section{Operation Alone}

Total hysterectomy and bilateral salpingooophorectomy is performed a week after diagnostic curettage. Before the operation is commenced the cervical canal is plugged and sutured to avoid any pieces of growth being expressed from the uterus into the operation area. The uterus is removed with the minimum of handling, in the usual way, the ovario-pelvic and round ligaments being severed as near as possible to the pelvic wall. The fimbriated ends of the Fallopian tubes may be ligatured to prevent shedding of growth from the lumen, though by many this step is not considered essential. Care is taken when finally removing the uterus that no part of the wound is touched by the cervix.

The results obtained by this method when there: has been no clinical evidence of gross spread from the uterus are excellent. Various authors' figures are all in the neighbourhood of 75 to 80 per cent. five-year cures (Dearnley, 1949; Rickford, 1949; see table). Recurrences, however, are not uncommon after this five-year period, and the majority occur both in these cases and those that die earlier, in the vaginal vault.

The operative mortality when the bad risks are treated other than by surgery is not more than I per cent.

\section{Pre-Operative Irradiation Followed by Surgery}

This method of treatment aims at 'sterilizing' the endometrial cavity prior to operation in order to minimize the risks of depositing active growth during its removal. Hundley et al. (r949) and others have been the main advocates of this system. Radium is inserted on one occasion six weeks before the hysterectomy, giving a dose of 5,000 mg. hours. Miller (1946) prefers radiotherapy as it - reduces any risk of spreading the disease during the manipulations of insertion of radium.
The disadvantage of this method is that it has been shown that the growth is not always inactivated in the endometrium and never if there is spread deep in the uterine wall or in the tubes or ovaries. Valuable time is thereby lost after the diagnosis has been made before radical treatment is carried out. The reported results for the small series of cases so far described are equal with those for primary operation.

\section{Radium Alone}

Professor Heyman (1947) perfected a technique in which the cavity of the uterus was completely filled with capsules of radium, thereby irradiating all surfaces of the growth.

It is used in all patients where the disease is clinically confined to the uterus whether they are physically fit for operation or not.

The results from this clinic are comparable to those by the two other methods of treatment so far described, e.g. 75 per cent. cure rate. Surgery is reserved for cases in which recurrence occurs, 22 hysterectomies being necessary in a series of 316 (approximately 7 per cent.) cases treated.

\section{Vaginal Hysterectomy}

This method of attack has recently been dese cribed by Professor Bastiaanse of Amsterdam. II I 5 cases there was no mortality and $\mathbf{7 2 . 2}$ per cento were alive and well after five years. By using this method it might perhaps be possible to treat surgically some of those. patients where a laparotomy was considered to be inadvisable. However, this author has found that it was not possible to remove one or both ovaries at the time of the operation in 33 cases (28.7 per cent.). This would appear a rather serious criticism of vaginal hysterectomy as a routine method.

Summarizing the results of these three main systems of treatment it will be seen that the results obtained when treating patients with disease clinically confined to the uterus are almost identical, in fact about three out of every four will survive. This does not give a true picture of the whole position.

A proportion of patients when they are first seen have gross evidence of advanced disease, either in the peritoneal cavity, large ovarian secondaries, metastases at the vulva or gross infiltration of the pelvic cellular tissues.

When the results for all cases seen are considered. it will be found that rather less than two out of three will survive after treatment.

Surgery is probably the better treatment when all factors are considered. It is found that even when there is a marked ovarian mass present, which would be quite incurable by intra-auterine radium, complete surgical removal is possible and 
may cure the patient. Again if a small intestinal secondary with adhesion to the uterus is found at laparotomy, it may be possible to resect the bowel and do the hysterectomy and still cure the patient; whereas radium in such a case might well have disastrous results. Patients who are unfit for surgery stand an excellent chance of cure if treated by the Stockholm method of packing the uterus with radium capsules. Unfortunately other methods of radium treatment do not give nearly such good results.

Finally, what of the 20 or 25 patients in every 100 who develop recurrence within the five years after operation, and the few who do so after this lapse of time?

The majority of these recurrences develop in the vaginal vault. Do these grow 'from disease left behind in the parametrium or deposited at the time of operation? Some have attempted to prevent this by post-operative radium at the vault of the vagina.

The answer may be that microscopic growth has spread into the lymphatics of the cardinal ligaments and has not, therefore, been excised during a simple total hysterectomy. This is not likely to happen unless hte lower third of the endometrium is involved. It might be possible by careful fractional curettage to decide when such a condition is present. If so these patients might well survive if a modified Wertheim operation were performed without removal of the iliac glands.

These are questions for the future but many surgeons are even now extending the limits of the operative treatment of carcinoma of the endometrium. It is obviously unwise for extensive excision to be undertaken unless the patient is reasonably fit and it can be shown conclusively that there is involvement of the lower part of the body of the uterus.

\section{BIBLIOGRAPHY}

BARNS, H. H. F. (1942), f. Obst. Gyn. Brit. Emp., 49, 173. BASTIAANSE, M. A. VAN B. (1952), Ibid., 59, 6 I I.

DEARNLEY, G. (1949), lbid., 56, 819.

HEYMAN, J. (1947), Brit. F. Radiol., 20, 85.

HUNDLEY, J. MASON, DIGGS, S. EVERETT, and KARDASH, T. (1949), Amer. F. Obst. Gynec., 57, 52.

MILLER, N. F., and HENDERSON, C. W. (1946), Ibid., 52, 894. PALMER, J. P., REINHARD, M. C., SADUGOR, M. G., and GOLTZ, H. L. (1949), Ibid., 58, 457.

QUENU, J., BECLERE, C., and LARPENT, R. (1952), Sem. Hop., Paris, 28, 2219.

RICKFORD, B. (1949), Ұ. Obst. Gyn. Brit. Emp., 56, 4r.

TAYLOR, H. C., and BECKER, W. F. (1947), Surg. Gynec. Obst., 84, 129.

"ORTHOBAN" BANDAGES are made from the highest grades of hygienic bleached cotton and have been supplied for many years to Orthopaedic Hospitals and Clinics both in this country and abroad. Made in widths from 1 in. to 16 in. and in lengths of $3,4,5$ and 6 yards.

The manufacturers of "ORTHOBAN" also make all grades of absorbent and nonabsorbent cotton wools, splint waddings and fast-dyed bacteriological cotton wool.

Quotations and samples on request from

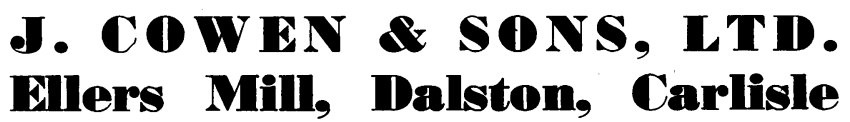

Established 1821 